\title{
WEIGHTED COMPOSITION OPERATORS AND DYNAMICAL SYSTEMS
}

\author{
R. K. SINGH AND BHOPINDER SINGH
}

\begin{abstract}
A.bstract. Let $X$ be a completely regular Hausdorff space, $E$ a Hausdorff locally convex topological vector space, and $V$ a system of weights on $X$. Denote by $C V_{b}(X, E)\left(C V_{o}(X, E)\right)$ the weighted space of all continuous functions $f: X \rightarrow E$ such that $v f(X)$ is bounded in $E$ (respectively, $v f$ vanishes at infinity on $X$ ) for all $v \in V$. In this paper, we give an improved characterization of weighted composition operators on $C V_{b}(X, E)$ and present a linear dynamical system induced by composition operators on the metrizable weighted space $C V_{\circ}(\mathbb{R}, E)$.
\end{abstract}

\section{Introduction and Preliminaries}

The contents of this paper pertain to the study of composition operators and their applications which have been the subject matter of study for the last 27 years or so . For details, we refer to Shapiro [11], Singh and Manhas [16], and the authors [20]. The weighted spaces of scalar-valued continuous functions, first introduced by $L$. Nachbin [6], were studied in details by W. H. Summers in his Doctoral Thesis [22], where as the weighted spaces of vector-valued functions were considered by J. B. Prolla $[7,8]$ and K. D. Bierstedt $[2,3]$.

Let us start with the following notations and definitions:

Let $X$ be a completely regular Hausdorff space and let $E$ be a Hausdorff locally convex topological vector space over $\mathbb{K}$, where the scalar field $\mathbb{K} \in\{\mathbb{R}, \mathbb{C})\}$. Then by $C(X, E)$ we mean the vector space of all continuous functions from $X$ into $E$, where as $c s(E)$ denotes the collection of all continuous seminorms on $E$. The symbol $B_{b}(E)$ (respectively, $B_{s}(E)$ ) stands for the space $B(E)$ of all operators on $E$ when it is equipped with the uniform (respectively, strong) operator topology, that is, the topology of uniform convergence on bounded (respectively, finite) subsets of $E$.

Definition 1. A weight $v$ on $X$ is a non-negative upper semicontinuous function on $X$. A system of weights on $X$ is the set $V$ of weights on $X$ such that $V$ is directed upward (or a Nachbin family [7]) and $V>0$ (i.e. for every $x \in X$, there is a $v_{x} \in V$ such that $\left.v_{x}(x)>0\right)$.

Received November 11, 1995.

1991 Mathematics Subject Classification. Primary 47B38, Secondary 46E40, 34C35.

Key words and phrases. Weighted composition operators, dynamical system, systems of weights, locally convex spaces, strong and uniform operator topologies . 
Definition 2.[22]: If $U$ and $V$ are two systems of weights on $X$ and for every $u \in U$, there is a $v \in V$ such that $u(x) \leq v(x)$ for all $x \in X$, then we write $U \leq V$. In case $U \leq V$ and $V \leq U$, we write $U \sim V$.

Definition 3. For a system $V$ of weights on $X$, let $C V_{o}(X, E)=\{f \in C(X, E)$ : $v f$ vanishes at infinity on $X$ for all $v \in V\} \& C V_{b}(X, E)=\{f \in C(X, E)$ : $v f(X)$ is bounded in $E$ for all $v \in V\}$. Both of these spaces are endowed with the Hausdorff locally convex topology (called the weighted topology) determined by the family $\left\{\|\cdot\|_{v, p}\right.$ : $v \in V$ and $p \in c s(E)\}$ of semi-norms, where $\|f\|_{v, p}=\sup \{v(x) p(f(x)): x \in X\}$, for all $f \in C V_{b}(X, E)$. Thus $C V_{b}(X, E)$ and $C V_{o}(X, E)$ are weighted spaces of vector-valued continuous functions. In case $E=\mathbb{K}$, we write $C V_{o}(X, \mathbb{K})$ as $C V_{o}(X)$. For more details and examples, we refer to Summers [23], Prolla [7], and Bierstedt [3].

Definition 4.[1] Let $Y$ be a topological vector space and let $\mathbb{R}$ have the usual topology. Then a function $\pi: \mathbb{R} \times Y \rightarrow Y$ is called a dynamical system on $Y$ if it satisfies the following:

(i) $\pi(0, y)=y$ for all $y \in Y$;

(ii) $\pi(a+b, y)=\pi(a, \pi(b, y))$ for all $a, b \in \mathbb{R}$ and $y \in Y$;

(iii) $\pi$ is continuous.

A dynamical system $\pi$ on $Y$ is called linear if

$$
\pi\left(a, \alpha y+\beta y^{\prime}\right)=\alpha \pi(a, y)+\beta \pi\left(a, y^{\prime}\right)
$$

holds for all $a \in \mathbb{R} ; \alpha, \beta \in \mathbb{K} ;$ and $y, y^{\prime} \in Y$.

The dynamical system $\pi$ induces two other continuous maps when one of the two variables is fixed. For each fixed $a \in \mathbb{R}, \pi^{\dot{a}}: Y \rightarrow Y$ defined as $\pi^{a}(y)=\pi(a, y)$ for all $y \in Y$ is called an action on $Y$, and for each fixed $y \in Y$, the function $\pi_{y}: \mathbb{R} \rightarrow Y$ defined as $\pi_{y}(a)=\pi(a, y)$ for all $a \in \mathbb{R}$ is called a motion through $y$.

In the next section, we present a characterization of weighted composition operators on $C V_{b}(X, E)$ which improves our earlier result from [19]. Following [21], we also obtain a linear dynamical system induced by the composition operators on the metrizable weighted space $C V_{o}(\mathbb{R}, E)$ in the last section.

\section{Weighted Composition Operators}

A weighted composition operator on the weighted space $C V_{a}(X, E)$, where $a \in\{o, b\}$, induced by the functions $w: X \rightarrow B(E)$ and $T: X \rightarrow X$ is a continuous linear transformation from $C V_{a}(X, E)$ into itself, which is denoted by $w C_{T}$ and has the following form:

$$
w C_{T} f(x)=w(x) f(T(x)) \quad \text { for all } x \in X \text { and } f \in C V_{a}(X, E) .
$$

In case $w(x)=I$, the identity operator on $E$, for all $x \in X$, the corresponding operator $w C_{T}$ on $C V_{a}(X, E)$ is called the composition operator and is denoted by $C_{T}$. On the 
other hand, when $T$ is the identity map on $X$, the corresponding operator $w C_{T}$ on $C V_{a}(X, E)$ is the multiplication operators $M_{w}$ on $C V_{a}(X, E)$.

A detailed study of composition operators on weighted spaces of scalar-valued continuous functions has been made by Singh and Summers in [21]. They proved that a selfmap $T$ on $X$ induces a composition operator $C_{T}$ on $C V_{b}(X)$ if and only if $T$ is continuous and $V \leq V o T$, where $V o T=\{v o T: v \in V\}$. In case of $C V_{o}(X), C_{T}$ is a composition operator induced by some selfmap $T$ on $X$ if and only if $T$ is continuous, $V \leq V o T$, and for every $v \in V, \varepsilon>0$ and compact subset $K$ of $X, T^{-1}(K) \cap\{x \in X: \bar{v}(x) \geq \varepsilon\}$ is compact. Similar type of results characterizing composition operators on weighted spaces of vector-valued continuous functions have been obtained by Singh and Manhas in [17]. Also, they have studied multiplication operators on weighted spaces in [14]. In [19], we have characterized weighted composition operators on $C V_{b}(X, E)$ for any locally convex space $E$ and for any system of weights $V$ on $X$. The following theorem is the main result in [19].

Theorem 2.1.[19] Let $w \in C\left(X, B_{b}(E)\right)$ and let $T$ be a continuous selfmap on $X$. Assume also that $w(X)$ is an equicontinuous subset of $B(E)$. Then $w C_{T}$ is a weighted composition operator on $C V_{b}(X, E)$ if and only if for each $v \in V$ and $p \in c s(E)$, there exists $u \in V$ and $q \in c s(E)$ such that $v(x) p(w(x) t) \leq u(T(x)) q(t)$ for all $x \in X$ and $t \in E$.

The proof of this result reveals that we have used the continuity of $w$ in the strong operator topology and so there is no need to take $w$ continuous in the uniform operator topology as a part of the hypothesis. In the following Theorem we will attempt to improve Theorem 2.1, where we donot require the selfmap $T$ to be continuous on whole of $X$. To see this, let us first note the following facts:

Let $w C_{T}$ be a weighted composition operator on $C V_{b}(X, E)$ and assume that $V$ consists of bounded weights on $X$. Then for any $t \in E$, the function $1_{t}$ defined by setting $1_{t}(x)=t$ for all $x \in X$ clearly belongs to $C V_{b}(X, E)$. Further, if $\left\{x_{\alpha}\right\}$ is a net in $X$ such that $x_{\alpha} \rightarrow x$ in $X$, then we have

$$
p\left[w\left(x_{\alpha}\right) t-w(x) t\right]=p\left[w C_{T} 1_{t}\left(x_{\alpha}\right)-w C_{T} 1_{t}(x)\right] \rightarrow 0
$$

for every $p \in c s(E)$ and $t \in E$. This implies that $w \in C\left(X, B_{s}(E)\right.$ ). (Note that $w$ is not necessarily continuous in the uniform operator topology. See also [5] for example). This continuity of $w$ shows that the set $N(w)=\{x \in X: w(x) \neq 0\}$ is open in $X$. Also, we shall see (in Theorem 2.2 below) that $T$ is continuous on $N(w)$. But $T$ is not necessarily continuous on $X \backslash N(w)$, because $w C_{T} f$ is zero on $X \backslash N(w)$ for every $f \in C V_{b}(X, E)$ even if $T$ is anyhow defined.

Theorem 2.2. Let $w$ be a $B(E)$-valued function on $X$ such that $w(X)$ is equicontinuous and let $T$ be a selfmap on $X$. Then the following conditions are sufficient for the pair $(w, T)$ to induce a weighted composition operator on $C V_{b}(X, E)$ :

(i) $w \in C\left(X, B_{s}(E)\right)$; 
(ii) $T$ is continuous on $N(w)$;

(iii) for every $v \in V$ and $p \in c s(E)$, there exists $u \in V$ and $q \in c s(E)$ such that $v(x) p(w(x) t) \leq u(T(x)) q(t)$ for all $x \in X$ and $t \in E$.

Further, if $V$ consists of bounded weights on $X$, then the conditions (i)-(iii) are also necessary for $(w, T)$ to induce a weighted composition coperator on $C V_{b}(X, E)$.

Proof. Since the sufficiency part is similar to that of the same part in Theorem 2.1, we need to prove that the conditions (i)-(iii) are necessary for the linear transformation $w C_{T}$ to be a weighted composition operator on $C V_{b}(X, E)$, under the assumption that weights are bounded. Suppose that $w C_{T}$ is a weighted composition operator on $C V_{b}(X, E)$. Then (i) holds (as noted above) and (iii) follows in a similar way as in Theorem 2.1. We proceed to prove (ii). For this, suppose on the contrary that there exists an $x_{o}$ in $N(w)$ at which $T$ is not continuous. Then there exists a net $\left\{x_{\alpha}\right\}_{\alpha \in A}$ in $N(w)$ such that $x_{\alpha} \rightarrow x_{0}$ but $T\left(x_{\alpha}\right)$ does not converge to $T\left(x_{0}\right)$. This implies that there exists an open neighbourhood $G$ of $T\left(x_{o}\right)$ such that for each $\alpha_{o} \in A, T\left(x_{\alpha}\right) \notin G$ for some $\alpha \geq \alpha_{o}$. Thus we can find a subnet $\left\{x_{\beta}\right\}$ of $\left\{x_{\alpha}\right\}$ such that $T\left(x_{\beta}\right) \notin G$. By Nachbin Lemma [6, page 69], there exists an $f \in C V_{o}(X)$ such that $0 \leq f \leq 1, f\left(T\left(x_{o}\right)\right)=1$ and $f(y)=0$ for all $y \notin G$. Let $t \in E$ such that $w\left(x_{o}\right) t \neq 0$, and choose $q \in \operatorname{cs}(E)$ such that $q\left(w\left(x_{o}\right) t\right)=1$. Define the function $f_{t}$ in $C V_{b}(X, E)$ by setting $f_{t}(x)=f(x) t$ for all $x \in X$. Then $w C_{T} f_{t} \in C V_{b}(X, E)$. But for each $\beta$, we have

$$
q\left[w C_{T} f_{t}\left(x_{\beta}\right)-w C_{T} f_{t}\left(x_{o}\right)\right]=q\left[w\left(x_{o}\right) t\right]=1 .
$$

This contradiction show that $T$ must be continuous on $N(w)$, and hence completes the proof.

Remark. (a) In the above theorem, we have used the boundedness of weighte only to prove that the condition (i) is necessary. In case $w(x)=I$ for all $x \in X$, this condition is trivially satisfied, and further when $E=\mathbb{C}$ our theorem reduces to [21, Theorem, 3.2].

(b) The conditions of the above theorem are not sufficient for the pair $(w, T)$ to induce a weighted composition operator on $C V_{o}(X, E)$, (see [21, page 307] for an example).

(c) When $T$ is the identity map on $X$, our theorem yields a corrected version of [14, Theorem 2.1] characterizing multiplication operators on $C V_{b}(X, E)$, (see also [15]).

(d) In case $V$ is the system of constant weights on $X$ and $E$ is a Banach space, $C V_{b}(X, E)$ is the Banach space $C_{b}(X, E)$ with the supremum norm. In this setting, composition operators and weighted composition operators have been studied by the second author in $[12,13]$.

\section{Dynamical Systems Induced by Composition Operators}

Throughout this section we shall take $E$ to be a Banach space with the norm $\|\cdot\|$ and $X=\mathbb{R}$ with the usual topology. Let $V$ be a system of weights on $\mathbb{R}$. Then $C V_{o}(\mathbb{R}, E)$ is a locally convex Hausdorff space (by definition 3 ). Now let $U$ be a countable set of weights 
on $\mathbb{R}$ such that $W=\{\alpha u: \alpha \geq 0, u \in U\}$ is a system of weights on $\mathbb{R}$ with $W \sim V$. Then the weighted space $C V_{0}(\mathbb{R}, E)$ is metrizable (and is normable if $U$ is finite), (cf. [22, page 18]).

For fixed $a \in \mathbb{R}$, define $T_{a}: \mathbb{R} \rightarrow \mathbb{R}$ as $T_{a}(x)=x+a$ for all $x \in \mathbb{R}$. Then $T_{a}$ is a. homeomorphism from $\mathbb{R}$ onto itself, and so by the remark following Theorem 2.3 in [21] or $\left[17\right.$, Remark 2.6] we see that $T_{a}$ induces a composition operator on $C V_{o}(\mathbb{R}, E)$ if and only if $V \leq V o T_{a}$. In [21, Theorem 2.6], Singh and Summers have obtained a linear dynamical system on the weighted (Banach) space $C V_{o}(\mathbb{R})$, where $V$ is the system generated by a single continuous weight on $\mathbb{R}$.

Now in the follwing theorem, we shall prove an analogue of this result on the metrizable weighted locally convex space $C V_{o}(\mathbb{R}, E)$ when $V$ is a system of bounded weights on $\mathbb{R}$.

Theorem 3.1. Let $V$ be a system of bounded weights on $\mathbb{R}$, and assume that $C V_{o}(\mathbb{R}, E)$ is metrizable. Consider the function $\pi: \mathbb{R} \times C V_{o}(\mathbb{R}, E) \rightarrow C(\mathbb{R}, E)$ defined by setting $\pi(a, f)=C_{T_{a}} f$ for every $a \in \mathbb{R}$ and $f \in{ }^{\cdot} C V_{o}(\mathbb{R}, E)$. Then the following statements are equivalent:

(i) $\pi$ is a linear dynamical system on $C V_{o}(\mathbb{R}, E)$;

(ii) $V \leq V o T_{a}$ for every $a \in \mathbb{R}$;

(iii) $C_{T_{a}}\left(C V_{o}(\mathbb{R}, E)\right) \subset C V_{b}(\mathbb{R}, E)$ for every $a \in \mathbb{R}$.

Proof. (i) $\Rightarrow$ (iii). If $\pi$ is dymanical system on $C V_{o}(\mathbb{R}, E)$, then $\pi$ maps $\mathbb{R} \times C V_{o}(\mathbb{R}, E)$ into $C V_{o}(\mathbb{R}, E)$. Thus (iii) holds.

(iii) $\Rightarrow$ (ii). The proof of this implication is similar to that of the same part of [21, Theorem 2.6].

(ii) $\Rightarrow$ (i). Suppose that $V \leq V o T_{a}$ for every $a \in \mathbb{R}$. Then by [17, Remark 2.6] each $C_{T_{a}}$ is a composition operator on $C V_{o}(\mathbb{R}, E)$. So $\pi$ is a function from $\mathbb{R} \times C V_{o}(\mathbb{R}, E)$ to $C V_{o}(\mathbb{R}, E)$ and clearly it satisfies the following properties:

$$
\begin{aligned}
\pi(0, f) & =f, \pi(a+b, f)=\pi(a, \pi(b, f)) \text { and } \\
\pi(a, \alpha f+\beta g) & =\alpha \pi(a, f)+\beta \pi(a, g) \quad \text { for every } a, b \in \mathbb{R},
\end{aligned}
$$

$f, g \in C V_{o}(\mathbb{R}, E)$ and $\alpha, \beta \in \mathbb{K}$.

Next to show that $\pi$ is jointly continuous, in view of [4, Theorem 1], it is enough to show that $\pi$ is separately continuous. For this, let $a \in \mathbb{R}$ and let $\left\{a_{n}\right\}$ be a sequence in $\mathbb{R}$ such that $a_{n} \rightarrow a$. We show that for fixed $f \in C V_{o}(\mathbb{R}, E), \pi_{f}\left(a_{n}\right) \rightarrow \pi_{f}(a)$ in $C V_{o}(\mathbb{R}, E)$. Let $v$ be an arbitrary weight on $\mathbb{R}$ and let $m_{v}=\sup \{v(x): x \in \mathbb{R}\}$. Then

$$
\begin{aligned}
\left\|\pi_{f}\left(a_{n}\right)-\pi_{f}(a)\right\|_{v}= & \sup \left\{v(x)\left\|f\left(x+a_{n}\right)-f(x+a)\right\|: x \in \mathbb{R}\right\} \\
\leq & m_{v} \sup \left\{\left\|f\left(x+a_{n}\right)-f(x+a)\right\|: x \in \mathbb{R}\right\} \\
& \rightarrow 0 \text { since } a_{n} \rightarrow a \text { and } f \in C(\mathbb{R}, E) .
\end{aligned}
$$


To see the continuity of $\pi$ in the second argument, let $\left\{f_{n}\right\}$ be a sequence in $C V_{o}(\mathbb{R}, E)$ such that $f_{n} \rightarrow f$ in $C V_{o}(\mathbb{R}, E)$. Then $\left\|f_{n}-f\right\|_{v} \rightarrow 0$ for every $v \in V$. We show that $\pi^{a}\left(f_{n}\right) \rightarrow \pi^{a}(f)$ in $C V_{o}(\mathbb{R}, E)$ for any fixed $a \in \mathbb{R}$. For this, let $v \in V$ be given. Then by (ii), there exists $u \in V$ such that $v(x) \leq u\left(T_{a}(x)\right)=u(x+a)$ for all $x \in \mathbb{R}$. Now

$$
\left\|\pi^{a}\left(f_{n}\right)-\pi^{a}(f)\right\|_{v}=\left\|C_{T_{a}}\left(f_{n}-f\right)\right\|_{v} \leq\left\|f_{n}-f\right\|_{u} \rightarrow 0 .
$$

Thus $\pi$ is a linear dynamical system on $C V_{o}(\mathbb{R}, E)$, and the proof is complete.

Remark. Let $E$ be a Banach space and $V$ be the system of all bounded weights on $\mathbb{R}$. Then it is not always true that each translation induces a composition operator (and hence a dynamical system) on the metrizable weighted space $C V_{o}(\mathbb{R}, E)$. This we shall see in the following examples:

(i) Let $v(x)=e^{-|x|}$ for all $x \in \mathbb{R}$ and let $V=\{\alpha v: \alpha>0\}$. Then for every $a \in \mathbb{R}$, $V \leq V o T_{a}$ since $v(x) \leq e^{|a|} \cdot v\left(T_{a}(x)\right)$ for all $x \in \mathbb{R}$, (cf. [10, Example 3.2]). In this case each translation induces a composition operator on $C V_{o}(\mathbb{R}, E)$.

(ii) Let $v(x)=e^{-x^{2}}$ for all $x \in \mathbb{R}$ and let $V\{\alpha v: \alpha>0\}$. Then $V \leq V_{0} T_{a}$ only when $a=0$. In this case, no non-identity translation induces composition operator on $C V_{o}(\mathbb{R}, E)$.

(iii) Let $V=\{\alpha v: \alpha>0\}$, where

$$
v(x)=\left\{\begin{array}{c}
e^{-x} \text { for } x \geq 0 \\
e^{-x^{2}} \text { for } x<0
\end{array}\right.
$$

Then only positive translations induce composition operators on $C V_{o}(\mathbb{R}, E)$.

Let $\ell=\left\{C_{T_{a}}: a \in \mathbb{R}\right\}$ be the family of all composition operators on the metrizable weighted space $C V_{o}(\mathbb{R}, E)$ induced by translations $T_{a}$ on $\mathbb{R}$. Then it satisfies the following properties for every $f \in C V_{o}(\mathbb{R}, E)$ :

$$
C_{T_{a}} f=f, C_{T_{a+b}} f=C_{T_{a}}\left(C_{T_{b}}\right) \quad \text { for } a, b \in \mathbb{R} \text { and } \lim _{a \rightarrow 0} C_{T_{a}} f=f .
$$

Thus $\ell$ is a $C_{o}$-group of composition operators on $C V_{o}(\mathbb{R}, E)$. In case $C V_{o}(\mathbb{R}, E)$ is also complete (cf. [3]), it can be shown in a similar way as in [18] by using a Corollary of the Banach Steinhass Theorem from [9, Theorem 2.6] that the $C_{o}$-group is locally equicontinuous.

\section{References}

[1] N. P. Bhatia and G. P. Szego, "Dynamical Systems: Stability theory and applications," Lecture Notes in Mathematics 35, Springer-Verlag, 1967.

[2] K. D. Bierstedt. "Gewichtete raume stetiger vektorwertiger funktionen und das injektive tensor produkt I(II)," J. Reine Angew. Math., 259(260), 1973, 186-210(133-146).

[3] - . "The Approximation-Theoretic localization of schwartz approximation property for weighted locally convex function spaces and some examples," Lecture Notes in Mathematics 843, Springer-Verlag (1981), 93-149. 
[4] P. Chernoff and J. Marsden, "On continuity and smoothness of group actions," Bull. Amer. Maith. Soc., 76(1970), 1044-1049.

[5] J. E. Jamison and M. Rajagopalan, "Weighted composition operator on $C(X, E)$," J. Operator Theory, 19(1988), 307-317.

[6] L. Nachbin, "Elements of approximation theory," Math. Studies 14, Van Nostrand, Princeton, 1967.

[7] J. B. Prolla, "Weighted spaces of vector valued continuous functions," Ann. Mat. Pura Appl., 89(1971), 145-157.

[8] _ - "Approximation of Vector-Valued functions," North-Holland Math Studies 25,Amsterdam, 1977.

[9] W. Rudin, Functional analysis, Tata Mcgraw-Hill, Inc., 1979.

[10] W. M. Ruess and W. H. Summers, "Minimal sets of almost periodic motions," Math. Ann., 276(1986), 145-158.

[11] J. H. Shapiro, "Composition operators and classical function theory," Springer-Verlag, 1993.

[12] Bhopinder Singh, "Composition operators on $C_{b}(X, E)$," Jndian J. Pure Appl. Math., 26(1995), 97-101.

[13] - and Kamalject Kour, "Weighted composition operators on $C_{b}(X, E)$.," Bull. Gauhati Univ. Math. Association, to appear.

[14] R. K. Singh and J. S. Manhas, "Multiplication operators and dynamical systems," J. Austral. Math. Soc., (Series A) 53(1992), 92-102.

[15] - "Corrigendum to multiplication operators and dynamical systems," J. Austral. Math. Soc.' (Series A) 58(1995), 142-142.

[16] _- "Composition operators on function spaces," North-Holland Maths Studies, 179, Amsterdam, 1993.

[17] — "Composition operators on weighted spaces of vector-valued continuous functions," Acto Sci. Math., (Szeged) 58(1993), 453-472.

[18] — , "Operators and dynamical systems on weighted function spaces," Math. Nachr., 169(1994), 279-285.

[19] R. K. Singh and Bhopinder Singh, "A characterization of weighted composition operators," Rockey Mountain J. Math., 23(1993), 1107-1114.

[20] — - "Compact weighted composition operators on spaces of continuous functions: A survey," Extracta, Math., 10(1995), 1-20.

[21] R. K. Singh and W. H. Summers, "Composition operators on weighted spaces of continuous functions," J. Austral Math. Soc., (Series A) 45(1988), 303-319.

[22] W. H. Summers, "Weighted locally convex spaces of continuous functions," Ph. D. Thesis, Louisiana State University, 1968.

[23] — , "A representation theorem for biequicontinuous completed tensor products of weighted spaces," Trans. Amer. Math. Soc., 146(1969), 121-131.

Department of Mathematics, University of Jammu, Jammu-180 004, India. 